\title{
Hyper-Geometric Distribution Software Reliability Growth Model with Imperfect Debugging
}

\author{
Rong-Huei Hou \& Sy-Yen Kuo \\ Department of Electrical Engineering \\ National Taiwan University \\ Taipei, Taiwan, R.O.C. \\ Email: sykuo@cc.ee.ntu.edu.tw
}

\author{
Yi-Ping Chang \\ Department of Business Mathematics \\ Soochow University \\ Taipei, Taiwan, R.O.C.
}

\begin{abstract}
Debugging actions during the test/debug phase of software development are not always performed perfectly. That is, not all the software faults detected are perfectly removed without introducing new faults. This phenomenon is called the imperfect debugging. The Hyper-Geometric Distribution software reliability growth Model (HGDM) was developed for estimating the number of software faults initially in a program. In this paper, we propose an extended model based on the HGDM incorporating the notion of imperfect debugging.
\end{abstract}

\section{Introduction}

Software Reliability Growth Models (SRGMs) have been studied by many authors [1]. Most of the SRGMs proposed are based on the assumption of perfect debugging. However, in reality, not all the software fault$s$ detected are perfectly removed without introducing new faults [2]. Most of the existing SRGMs do not take this into account, and only a few researchers extended the SRGMs considering imperfect debugging [3-8].

The Hyper-Geometric Distribution software reliability growth Model (HGDM) for estimating the number of initial software faults, first proposed by Tohma et al. [9], is shown to be attractive. Tohma et. al. [1012], Jacoby et. al. [13-14], and Hou et. al. [15-16] have made a series of studies on the HGDM recently. In this paper, we propose an extended model based on the HGDM incorporating the notion of imperfect debugging. Furthermore, to make the proposed model more realistic and practical, we consider the situation where there is learning implicitly in the fault removal process. The growth curve of the cumulative number of discovered faults and a measure of software reliability for the HGDM with imperfect debugging are investigated. In addition, the relationship between the proposed model and the Goel-Okumoto NHPP model with imperfect debugging is discussed. Experiments have been performed by using two real test/debug data sets, and the results show the proposed model fits the data sets satisfactorily.

\section{Review of HGDM}

In this section, we briefly review the HyperGeometric Distribution software reliability growth Model (HGDM) [9-14]. In general, a program is assumed to have $m$ faults initially before the test/debug phase starts. The collection of test operations performed in a day or a week is called a "test instance". Test instances are denoted by $t_{i}, i=1,2, \ldots, n$ in accordance with the order of applying them. The "sensitivity factor", $w_{i}$, represents how many faults are newly discovered or rediscovered during the application of test instance $t_{i}$. Some of the faults detected by $t_{i}$ may have been detected previously by the application of $t_{1}, t_{2}, \ldots, t_{i-1}$.

Let $C_{i-1}$ be the number of faults already detected so far by $t_{1}, t_{2}, \ldots, t_{i-1}$ and $x_{i}$ be the number of faults newly detected by $t_{i}$. Then, some of the $w_{i}$ faults may be those already counted in $C_{i-1}$, and the remaining $w_{i}$ faults account for the newly detected faults. With the assumption that new faults will not be introduced into the program while correcting is being performed, the conditional probability $P\left(N_{i}=x_{i} \mid m, w_{i}, C_{i-1}\right)$ can be formulated as:

$P\left(N_{i=x_{i}} \mid m, w_{i}, C_{i-1}\right)=\frac{\left(\begin{array}{c}m-C_{i-1} \\ x_{i}\end{array}\right)\left(\begin{array}{c}C_{i-1} \\ w_{i}-x_{i}\end{array}\right)}{\left(\begin{array}{l}m \\ w_{i}\end{array}\right)}$, 
where $\max \left(0, w_{i}-C_{i-1}\right) \leq x_{i} \leq \min \left(w_{i}, m-C_{i-1}\right)$ for all $i \geq 0, C_{i-1}=\sum_{k=1}^{i-1} x_{k}, C_{0}=0, \quad x_{0}=0$ and $x_{k}$ is an observed instance of $N_{k}$. The expected value of $C_{i}$ denoted by $E C_{i}$ is [9-14]

$$
\left\{\begin{array}{l}
E C_{0}=0, \\
E C_{i}=m\left[1-\prod_{j=1}^{i}\left(1-p_{j}\right)\right], i=1,2, \ldots, n,
\end{array}\right.
$$

where $p_{i}=w_{i} / m$. Although various functions for $p_{i}$ have been presented, the linear function $p_{i}=e i+f$ usually gives satisfactory results [11-12].

From Eq.(2), $\left(E C_{i}-E C_{i-1}\right) /\left(m-E C_{i-1}\right)=p_{i}$ represents the ability of test workers to detect new faults at the application of $t_{i}$. If the test is completely random, that is, test data are taken randomly from the input space of the program, $p_{i}$ will be a constant. In following sections, we propose an extended model under the assumption that $p_{i}$ is a constant.

\section{HGDM with Imperfect Debugging}

In Section 2 we reviewed the HGDM, which assumes the detected faults are instantly and perfectly corrected without introducing any new faults. However, this assumption is not always valid and can be modified to be:

(i) When the detected faults are removed, it is possible to introduce new faults.

(ii) When a fault is newly discovered during the application of $t_{i}$, removal of the fault is instantaneous and the following may occur:

(a) the fault is corrected with probability $1-\theta_{i}$;

(b) a new fault is introduced with probability $\theta_{i}$

On the basis of above assumption, we propose an extended model based on the HGDM incorporating the notion of imperfect debugging in the following.

\subsection{Mean value function of the HGDM with imperfect debugging}

Let $m_{0}=m$ be the number of initial faults in a program before the test/debug phase. Suppose there are $x_{1}$ new faults discovered by $t_{1}$. Since the detected faults are instantly corrected and the fault introduction rate during the application of $t_{1}$ is $\theta_{1}$, there are $\theta_{1} x_{1}$ faults newly introduced during the removal of the detected faults. Therefore, the number of faults including initial faults and the faults newly introduced by $t_{1}$ are $m_{1}=m_{0}+\theta_{1} x_{1}=m+\theta_{1} x_{1}$. Suppose there are $x_{2}$ new faults discovered by $t_{2}$. Since the fault introduction rate during the application of $t_{2}$ is $\theta_{2}$, there are $\theta_{2} x_{2}$ faults newly introduced during the removal of the detected faults. Therefore, the number of faults including initial faults and all the faults introduced so far by $t_{1}$ and $t_{2}$ are $m_{2}=m_{1}+\theta_{2} x_{2}=m+\theta_{1} x_{1}+\theta_{2} x_{2}$.
Let $m_{i}$ be the number of faults including the initial faults and all the faults already introduced so far by $t_{1}, t_{2}, \ldots, t_{i}$. The HGDM with imperfect debugging can be derived as follows.

$$
\begin{aligned}
m_{0}= & m ; \\
m_{1}= & m_{0}+\theta_{1} N_{1}=m+\theta_{1} N_{1} ; \\
& \ldots \ldots \\
m_{n}= & m+\sum_{i=1}^{n} \theta_{i} N_{i} .
\end{aligned}
$$

Therefore, Eq.(1) can be modified to be

$$
P\left(N_{i}=x_{i} \mid m_{i-1}, w_{i}, C_{i-1}\right)=\frac{\left(\begin{array}{c}
m_{i-1}-C_{i-1} \\
x_{i}
\end{array}\right)\left(\begin{array}{c}
C_{i-1} \\
w_{i}-x_{i}
\end{array}\right)}{\left(\begin{array}{c}
m_{i-1} \\
w_{i}
\end{array}\right)}
$$

where $\max \left(0, w_{i}-C_{i-1}\right) \leq x_{i} \leq \min \left(m_{i-1}-C_{i-1}, w_{i}\right)$. Under the assumption that the test is completely random, we have

$$
\frac{w_{i}}{m_{i-1}}=p \quad \forall i=1,2, \ldots, n, \text { and } 0 \leq p \leq 1 .
$$

For convenience, let

$$
C_{i}^{*}=\sum_{k=1}^{i}\left(1-\theta_{k}\right) N_{k}, \quad i=1,2, \ldots, n .
$$

Since $m_{i-1}=m+\sum_{k=1}^{i-1} \theta_{k} N_{k}$, we have

$$
\begin{aligned}
E N_{i} & =E\left(E\left(N_{i} \mid N_{i-1}\right)\right)=E\left[\left(m_{i-1}-C_{i-1}\right) p\right] \\
& =p\left(m-E C_{i-1}^{*}\right), \quad i=1,2, \ldots, n .
\end{aligned}
$$

From Eqs.(6) and (7), we have

$$
E C_{i}^{*}=\left\{1-\left(1-\theta_{i}\right) p\right\} E C_{i-1}^{*}+m p\left(1-\theta_{i}\right) .
$$

The solution to this difference equation is [10]

$$
E C_{i}^{*}=m\left[1-\prod_{k=1}^{i}\left\{1-\left(1-\theta_{k}\right) p\right\}\right], \quad i=1,2, \ldots, n .
$$

Therefore, $E C_{i}$ (i.e., $\sum_{k=1}^{i} E N_{k}$ ) for the HGDM with imperfect debugging is

$$
\left\{\begin{array}{l}
E C_{1}=m p, \\
E C_{i}=m p\left[1+\sum_{j=2}^{i} \prod_{k=1}^{j-1}\left\{1-\left(1-\theta_{k}\right) p\right\}\right], i=2, \ldots, n .
\end{array}\right.
$$

\subsection{Characteristics of fault introduction rate}

The fault introduction rate $\theta_{i}$ is the probability of fault introduction during the application of $t_{i}$, and it should satisfy the condition: $0 \leq \theta_{i}<1$ for all $i \geq 1$. If $\theta_{i}$ equals zero for all $i \geq 1$, it is equivalent to the perfect debugging case. If $\hat{\theta}_{i}$ is a constant for all $i \geq 1$, it assumes the probability of introducing a software fault 
is a constant. However, the assumption of $\theta_{i}$ being a constant is not realistic. Since the skill to remove software faults will improve with the experience, the effect of learning in the progress of removing faults should be taken into consideration.

Traditionally, the "decreasing exponential curve" or the "decreasing $S$-shaped curve" as shown in Figure 1 is commonly used to interpret the human learning process under the imperfect debugging environment. The decreasing exponential learning curve assumes the fault introduction rate decreases faster at the initial application of test instances than at later stages. The decreasing S-shaped learning curve assumes that the fault introduction rate decreases slowly at the beginning stages of testing because the skill to remove faults is not experienced initially; at the subsequent stages, the rate decreases quickly because the skill becomes versed; at the later stages, the rate again decreases slowly.

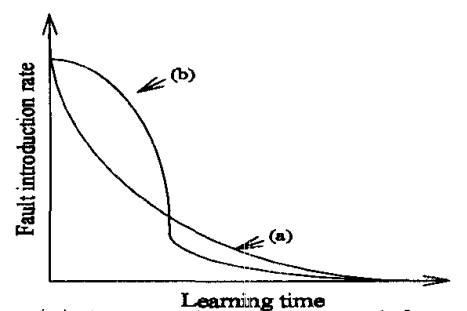

Figure 1. (a) Decreasing exponential curve; (b) decreasing $\mathbf{S}$-shaped curve.

A continuous function $\theta(t)$ which can describe either the decreasing S-shaped curve or the decreasing exponential curve is given by

$$
\theta(t)=\frac{1}{1+e^{a t+b}}, \quad a>0, t \geq 0 .
$$

Through simple calculation, the following may be obtained:

(i) If $b<0, \theta(t)$ is a decreasing $\mathbf{S}$-shaped curve.

(ii) If $b \geq 0, \theta(t)$ is a decreasing exponential curve.

Based on above discussion for the continuous function $\theta(t)$, a discrete function $\theta_{i}$ can be defined as

$$
\theta_{i}=\theta(i)=\frac{1}{1+e^{a i+b}}, \quad i=1,2, \ldots, n .
$$

Thus, we can use $\theta_{i}$ to interpret a discrete decreasing S-shaped or exponential learning curve.

\subsection{Estimation of parameters by least squares method}

The parameters of the HGDM with imperfect debugging can be estimated by the least squares method. For the given sample observations, the sum of squares of errors is

$$
S\left(m, p, \theta_{i}\right)=\sum_{i=1}^{n}\left(C_{i}-E C_{i}\right)^{2},
$$

where $E C_{i}$ is given by Eq.(9) and $\theta_{i}=1 /\left(1+e^{a i+b}\right)$. The IMSL MATH/LIBRARY Subroutine UNLSF [17] is used for the minimization of Eq.(12), and the estimates of these four parameters $m, p, a$, and $b$ can be obtained.

\section{Growth Curve of $E C_{i}$ and a Measure of Soft- ware Reliability}

\subsection{A finite-fault category}

A classification scheme was proposed by Musa and Okumoto [18] for software reliability growth models. The following theorem shows the HGDM with imperfect debugging belongs to the finite fault category.

Theorem 1. Assuming $\theta_{i}=1 /\left(1+e^{a i+b}\right)$ for $i \geq 1$, the upper bound of $E C_{i}$ is finite.

Proof: Since $\theta_{i}$ is decreasing in $i$, we have

$$
\begin{aligned}
\lim _{i \rightarrow \infty} E C_{i} & \leq \lim _{i \rightarrow \infty} m p\left\{1+\sum_{j=2}^{i} \prod_{k=1}^{i-1}\left[1-\left(1-\theta_{1}\right) p\right]\right\} \\
& =\lim _{i \rightarrow \infty} \frac{m}{1-\theta_{1}}\left\{1-\left[1-\left(1-\theta_{1}\right) p\right]^{i}\right\}=\frac{m}{1-\theta_{1}}
\end{aligned}
$$

Moreover, since $E C_{i}$ is increasing in $i$; the upper bound of $E C_{i}$ is finite.

\subsection{Growth curve of $E C_{i}$}

Since $E C_{i}$ is increasing in $i$, we have the following definition [15].

Definition 1. $E C_{i}$ is an exponential curve if $E C_{i+1}+$ $E C_{i-1}-2 E C_{i}<0$ for all $i \geq 1$.

Theorem 2. Suppose the test is completely random in the test/debug phase, i.e., $p_{i}=p$ for all $i \geq 1$. The growth curve of $E C_{i}$ shows an exponential growth curve no matter what $\theta_{i}$ is.

Proof: For all $i \geq 1$, we have

$$
\begin{aligned}
& E C_{i-1}+E C_{i+1}-2 E C_{i}=E N_{i+1}-E N_{i} . \\
& =-m p^{2}\left(1-\theta_{i}\right) \prod_{k=1}^{i-1}\left[1-\left(1-\theta_{k}\right) p\right]<0 .
\end{aligned}
$$

Theorem 2 indicates if $p_{i}$ is a constant, $E C_{i}$ shows an exponential curve no matter what $\theta_{i}$ is. In other words, if the growth curve of $E C_{i}$ is not an exponential curve, $p_{i}$ is not a constant.

\subsection{A measure of software reliability}

The term $m_{i}-C_{i}$ means the number of remaining faults after the application of $t_{i}$. Thereore, a measure of software reliability is defined as 
$R_{i}=1-\frac{m_{i}-C_{i}}{m}=\frac{\sum_{j=1}^{i}\left(1-\theta_{j}\right) N_{j}}{m}, i=0,1,2, \ldots, n$.

Note that $R_{i}$ is increasing in $i$ and $0 \leq R_{i} \leq 1$ for all $i$. The expected value of $R_{i}$ is

$$
E R_{i}=E\left[\frac{\sum_{j=1}^{i}\left(1-\theta_{j}\right) N_{j}}{m}\right]=\frac{E C_{i}^{*}}{m},
$$

where $C_{i}^{*}$ is defined in Eq.(6). It is obvious that $E R_{i}$ is increasing in $i$, and then we have the following definition [15].

\section{Definition 2.}

(i) $E R_{i}$ is an exponential curve if $E R_{i+1}+E R_{i-1}-$ $2 E R_{i}<0$ for all $i \geq 1$;

(ii) $E R_{i}$ is an $\mathrm{S}$-shaped curve if there exists a finite integer $I>1$ such that $E R_{i+1}+E R_{i-1}-2 E R_{i} \geq$ 0 for all $i<I$ and $E R_{i+1}+E R_{i-1}-2 E R_{i}<0$ for all $i \geq I$.

From Eq.(13), we have

$$
E R_{i+1}+E R_{i-1}-2 E R_{i}=\frac{E C_{i+1}^{*}+E C_{i-1}^{*}-2 E C_{i}^{*}}{m} \text {. }
$$

Since $\theta_{i}=1 /\left(1+e^{a i+b}\right)$, from Eq. (8), we have

$$
E C_{i}^{*}=m\left[1-\prod_{k=1}^{i}\left\{1-p /\left(1+e^{-a k-b}\right)\right\}\right], i=1,2, \ldots, n .
$$

Based on Eqs.(14) and (15), the following theorem of the growth curve of $E R_{i}$ can be obtained similarly [15].

Theorem 3. Assuming $\theta_{i}=1 /\left(1+e^{a i+b}\right)$, we have:

(i) if $1 \geq p>e^{-(2 a+b)\left(e^{a}-1\right)}$, then $E R_{i}$ is an exponential curve;

(ii) if $0 \leq p \leq e^{-(2 a+b)\left(e^{a}-1\right)}$, then $E R_{i}$ is an Sshaped curve.

\section{Precise Relationship to Goel-Okumoto Mod- el with Imperfect Debugging}

In this section, under the assumption of $\theta_{i}=\theta$ for all $i \geq 1$, the mathematical relationship between the mean value function of the HGDM with imperfect debugging and that of the Goel-Okumoto NHPP Model with imperfect debugging [5] is shown.

The mean value function $D(i)$ of the GoelOkumoto model with imperfect debugging is [5]:

$$
D(i)=\frac{m}{1-\beta}\left[1-e^{-(1-\beta) \phi i}\right]
$$

where $m$ is the number of initial faults in a program, $\beta$ is the fault introduction rate $(0 \leq \beta<1)$, and $\phi$ is the fault detection rate $(\phi>0)$.

If $\theta_{i}$ is a constant, from Eq.(9) we have

$$
E C_{i}=\frac{m}{1-\theta}\left\{1-e^{i \ln [1-(1-\theta) p]}\right\}
$$

Comparing Eq.(16) with Eq.(17), since both $\theta$ and $\beta$ are fault introduction rates, we can let

$$
\theta=\beta \text { and } p=\frac{1}{1-\beta}\left[1-e^{-(1-\beta) \phi}\right] .
$$

Since $0 \leq\left(1-e^{-(1-\beta) \phi}\right) /(1-\beta) \leq 1$ if and only if $0 \leq \phi \leq 1$, the relationship between the mean value functions of these two models is given by Eq.(18) when $0 \leq \phi \leq 1$

\section{Numerical Examples and Data Analysis}

To validate the proposed model, two software fault data sets are performed. The sum of squares of errors $S S E=\sum_{i=1}^{n}\left(C_{i}-\widehat{C}_{i}\right)^{2}$ is adopted as the evaluation criterion. From Eq.(13), two measures related to $E R_{i}$, the observed software reliability $R 1_{i}$ and the estimated software reliability $R 2_{i}$, are defined in the following,

respectively.
$R 1_{0}=0$, and $R 1_{i}=\frac{\sum_{j=1}^{i}\left(1-\widehat{\theta_{j}}\right) x_{j}}{\widehat{m}}, i=1,2, \ldots, n ;$
$R 2_{0}=0$, and $R 2_{i}=\frac{\sum_{j=1}^{i}\left(1-\widehat{\theta_{j}}\right) \widehat{E N_{j}}}{\widehat{m}}, i=1,2, \ldots, n$.

\subsection{First Data Set}

The first test/debug data set is presented in [19]. It is the collection of the cumulative number of discovered faults for the 81 test instances. The cumulative number of discovered faults up to the test instance $t_{81}$ is 460 .

The least squares estimates of parameters of the HGDM with imperfect debugging are $\widehat{m}=366.5, \widehat{p}=$ $0.0295, \widehat{a}=0.2806, \widehat{b}=-4.0461$. The sum of squares of errors $S S E$ is 6567.9 . Therefore, the number of faults introduced during the observation period as a result of imperfect debugging is approximately 93 .

The observed and estimated growth curves of the cumulative number of detected faults are shown in Figure 2. It can be seen that the estimated growth curve fits the data nicely. Since $\hat{b}=-4.05<0, \theta_{i}$ is a decreasing $\mathrm{S}$-shaped curve as shown in Figure 3 . The curves of $R 1_{i}$ and $R 2_{i}$ are plotted in Figure 4. Obviously, the curve of $R 2_{i}$ is an $\mathrm{S}$-shaped cure. In fact, the curve of $R 2_{i}$ being $S$-shape can be easily determined by Theorem 3 instead of burdensome plotting.

The least squares estimates of parameters of the HGDM are $\widehat{m}=545.67$ and $\widehat{p}=0.0239$, and the sum of squares of errors $S S E$ is 8181.18. Based on $S S E$, the HGDM with imperfect debugging fits the data more accurately than the HGDM.

\subsection{Second Data Set}

This data is presented in [20]. It is the collection of the cumulative number of discovered faults for the 
25 test instances. The least squares estimates of the parameters of the HGDM with imperfect debugging are $\widehat{m}=135.97, \widehat{p}=0.1291, \widehat{a}=0.9401, \widehat{b}=11.0243$. The sum of squares of errors $S S E$ is 766.1. Since all the values of $\theta_{i}, i=1,2, \ldots, 25$ tend to zero, the number of faults introduced during the fault removal process is approximately 0 .

The observed and estimated growth curves of the cumulative number of detected faults are shown in Figure 5 . The estimated growth curve fits the data satisfactorily. Since $\widehat{b}=11.0243>0, \theta_{i}$ is a decreasing exponential curve as shown in Figure 6. Moreover, since all the values of $\theta_{i}$ are negligible, the fault removal process can be regarded to be perfect debugging. Accordingly, the proposed model can be reduced to the HGDM. The curves of $R 1_{i}$ and $R 2_{i}$ are plotted in Figure 7 . Similarly, the curve of $R 2_{i}$ being exponential shape can be easily determined by Theorem 3 .

The least squares estimates of parameters of the HGDM are $\widehat{m}=135.98$ and $\widehat{p}=0.129$, and the sum of squares of errors $S S E$ is 766.1. Obviously, the HGDM with imperfect debugging is equivalent to the HGDM for this data set. Thus, we can still apply the HGDM with imperfect debugging even though the fault removal process is perfect debugging.

\section{Conclusions}

In this paper, we propose an extended model based on the HGDM by elimination of the assumption that the detected faults in a program can be perfectly removed. To make the proposed model more realistic and practical, we consider the situation where there is learning in the fault removal process. The experimental results show the HGDM with imperfect debugging can fit the data sets satisfactorily. Moreover, we can apply the HGDM with imperfect debugging even though the fault removal process is perfect. The growth curve of the cumulative number of discovered faults is investigated. The relationship between the proposed model and the Goel-Okumoto model with imperfect debugging is also discussed.

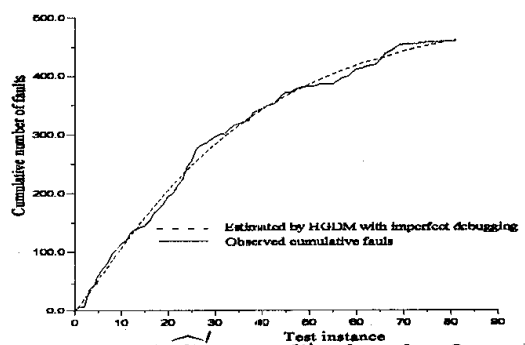

Figure 2. Fitness of $\widehat{C}_{i}^{\prime} s$ to $C_{i}^{\prime} s$ for the first data set.

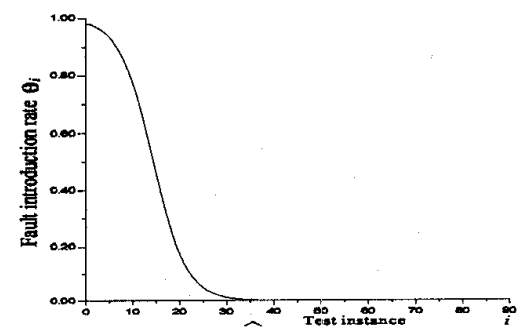

Figure 3. Curve of $\hat{\theta}_{i}$ for the first data set.

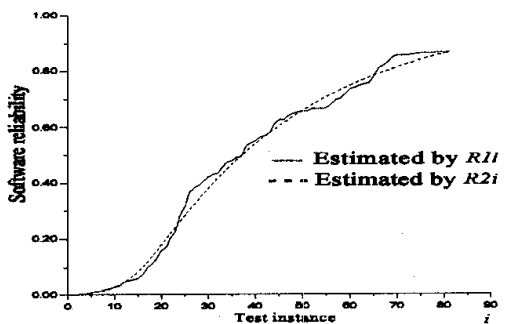

Figure 4. Curves of $R 1_{i}$ and $R 2_{i}$ for the first data set.

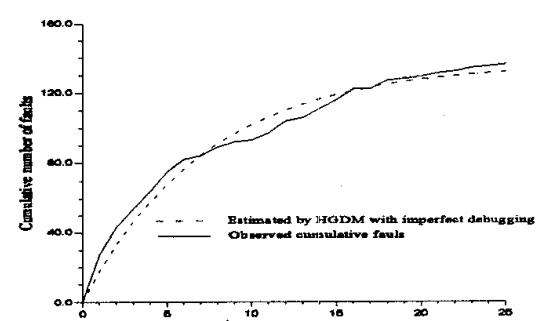

Figure 5. Fitness of ${\widehat{C_{i}}}_{i}^{\prime} s$ to $C_{i}^{\prime 0} s$ for second data set.

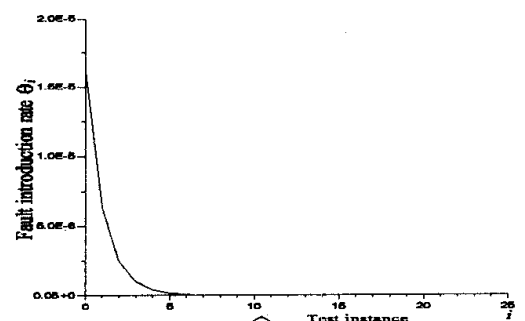

Figure 6. Curve of $\widehat{\theta}_{i}$ for second data set.

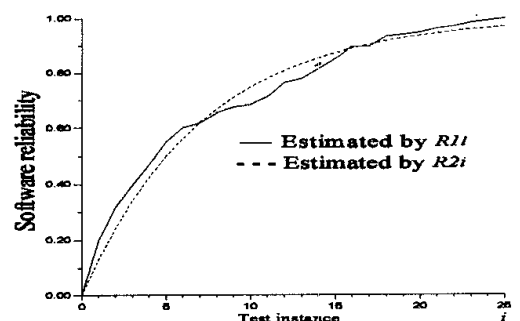

Figure 7. Curves of $R 1_{i}$ and $R 2_{i}$ for second data set.

Acknowledgment. We would like to express our gratitude for the support of the National Science Council, Taiwan, R.O.C., under Grant NSC 84-0408E002-008. Reviewers' comments are also highly appreciated. 


\section{References}

[1] C. V. Ramamoorthy and F. B. Bastani, "Software Reliability - Status and Perspectives", IEEE Trans. Software Engineering, Vol. 8, No. 4, pp. 354-371, 1982.

[2] B. Littlewood, "Theories of Software Reliability: How Good Are They and How Can They Be Improved?", IEEE Trans. Software Engineering, Vol. 6, No. 5, pp. 489-500, 1980.

[3] U. Sumita and J. G. Shanthikumar, "A Software Reliability Model with Multiple-Error Introduction \& Removal", IEEE Trans. Reliability, Vol. R-35, No. 4, pp. 459-462, 1986.

[4] M. Ohba and X. M. Chou "Does Imperfect Debugging Affect Software Reliability Growth?", Proc. 11th Int. Conf. Software Engineering, pp. 237-244, 1989.

[5] P. K. Kapur and R. B. Garg, "Optimum Software Release Policies for Software Reliability Growth Models under Imperfect Debugging", R.A.I.R.O., Vol. 24, No. 3 pp. 295-305, 1990.

[6] K. Tokunoh, S. Yamada, and S. Osaki, "A Markovian Imperfect Debugging Model for Software Reliability Measure", IEICE Trans. Fundmentals, Vol. E75-A, No. 11, pp. 1590-1596, 1992.

[7] P. K. Kapur, P. S. Grover and S. Younes, "Modeling an Imperfect Debugging Phenomenon with Testing Effort", Proc. Int. Symposium on Software Reliability Engineering, Monterey, California, pp. 178-183, November 1994.

[8] P. Zeephongsekul, G. Xia and S. Kumar, "Software-Reliability Growth Model: PrimaryFailures Generate Secondary-Faults Under Imperfect Debugging", IEEE Trans. Reliability, Vol. R-43, No. 3, pp. 408-413, 1994.

[9] Y. Tohma, K. Tokunaga, S. Nagase, and Y. Murata, "Structural Approach to the Estimation of the Number of Residual Software Faults Based on the Hyper-Geometric Distribution", IEEE Trans. Software Engineering, Vol. 15, No. 3, pp. $345^{-}$ 355, March 1989.

[10] Y. Tohma, R. Jacoby, Y. Murata, and M. Yamamoto, "Hyper-Geometric Distribution Model to Estimate the Number of Residual Software Faults", Proc. COMPSAC-89, Orlando, pp. 610617, September 1989.

[11] Y. Tohma, H. Yamano, M. Ohba, and R. Jacoby, "Parameter Estimation of the Hyper-Geometric
Distribution Model for Real Test/Debug Data", Proc. Int. Symposium on Software Reliability Engineering, Austin, Texas, May 17-18, pp. 28-34, 1991.

[12] Y. Tohma, H. Yamano, M. Ohba, and R. Jacoby, "The Estimation of Parameters of the Hypergeometric Distribution and Its Application to the Software Reliability Growth Model", IEEE Trans. Software Engineering, Vol. SE-17, No. 5, pp. 483-489, May 1991.

[13] R. Jacoby and Y. Tohma, "The Hyper-Geometric Distribution Software Reliability Growth Model (HGDM): Precise Formulation and Applicability", Proc. COMPSAC90, Chicago, pp. 13-19, October 1990.

[14] R. Jacoby and Y. Tohma, "Parameter Value Computation by Least Square Method and Evaluation of Software Availability and Reliability at Service-Operation by the Hyper-Geometric Distribution Software Reliability Growth Model (HGDM)", Proc. 13th Int. Conf. Software Engineering, pp. 226-237, 1991.

[15] R. H. Hou, S. Y. Kuo, and Y. P. Chang, "Applying Various Learning Curves to Hyper-Geometric Distribution Software Reliability Growth Model", Proc. Int. Symposium on Software Reliability Engineering, Monterey, California, pp. 7-16, November 1994.

[16] R. H. Hou, I. Y. Chen, Y. P. Chang, and S. Y. Kuo, "Optimal release policies for hypergeometric distribution software reliability growth model with scheduled delivery time", The AsiaPacific Software Engineering Conference, Tokyo, pp. 445-452, December 1994.

[17] IMSL MATH/LIBRARY, FORTRAN Subroutines for Mathematical Applications, 1991.

[18] J. D. Musa, A. Iannino, and K. Okumoto, "Software Reliability - Measurement, Prediction, Application", McGraw-Hill, INc., 1987.

[19] K. Kanoun, M. R. Bastos Martini, and J. Moreira de Souza, "A Method for Software Reliability Analysis and Prediction. Application to the Tropico-R Switching System", Research Report from LAAS-CNRS, France, 1989.

[20] R. Jacoby and Y. Tohma, "Note on the Application of the Hyper-Geometric Distribution to Estimate the Total Number of Faults Initially Resident in a Software Under Test", IECE Technical Report, FTS88-9, pp. 51-58, May 1988. 